\title{
Photometric and Polarimetric Observations of AGB and Post AGB Stars
}

\author{
N.D. Melikian
}

Byurakan Astrophysical Observatory. Ashtarak Aragatsotn District. Byurakan. Armenia

C. Magnan

GRAAL - CC 72 University de Montpellier II. 34095 Montpellier Cedex 05. France

\author{
A.A. Karapetian \\ Byurakan Astrophysical Observatory. Ashtarak Aragatsotn District. \\ Byurakan. Armenia
}

\section{J.M. Sarkissian}

CSIRO Parkes Observatory. PO Box 276. Parkes NSW 2870. Australia

\begin{abstract}
Photometric and polarimetric observations of Long-Period Variables (LPVs), carried out at Byurakan Observatory over the last few years, have yielded some interesting results. In particular, one of the important results obtained for Mira type variables is that there is a certain correlation between brightness and polarization: the maximum degree of polarization is midway on the increasing branch of the light curve and in all probability its change has a periodic character. More recently an important result has been obtained for a post AGB star SAO 124414. The polarimetric and photometric observations of this star were carried out over a period of 3 years. At an almost constant brightness, strong, irregular changes of light polarization were observed in three bands (BVR) of the spectrum. The analysis of the observations does not show periodicity in changes of polarization. In this report the observational results for SAO 124414 are presented. In the study of the evolution of red giants and supergiants, future observations of this star taken simultaneously in optical and radio wavelengths, could be very significant.
\end{abstract}

\section{Introduction}

The study of AGB and Post-AGB stars is very important in the investigations of the evolution of red giants and supergiants; it is a critical stage in their evolution. These stars, as precursors of planetary nebulae, exhibit high rates of mass loss and eject material in the form of a slow, dusty molecular wind. The post-AGB phase of evolution, when stars leave the red giant region eventually to become white dwarf (WD) remnants, is characterized by a series of intriguing events and 
diversities. The beginning of the post-AGB phase is conventionally set at the time of cessation of the high mass-loss rate episode $10^{-5} \mathrm{M} \odot /$ year, which has almost completely stripped the star of its hydrogen-rich envelope.

At Byurakan Observatory, an intensive photometric and polarimetric monitoring has been carried out over the last 6 years on about 40 AGB and post-AGB stars. Some interesting results have been obtained during these observations. It has been shown, that the degree of light polarization has a periodic character with the maximum being halfway on the increasing branch of the light curve. During the photographic observations of a Mira type long period variable star, Y Ori, a nebulosity was detected near its maximum brightness in red light only (Melikian et al. 1988; Melikian\&Jakubov 1995): observations at the same time in the $U$ and B bands of the spectra don't show any trace of nebulosity.

As pointed out above, the post AGB phase of evolution is characterized by a series of interesting events, one of which has been detected during the photometric and polarimetric observations of a post AGB star SAO 124414 (Melikian et al. 2000). In this report, the result of these observations is presented.

\section{Observations}

The present photometric and polarimetric observations were carried out over a 3 year period (1996-1998). The observations were performed with the photopolarimeter attached to the AZT-14 $50 \mathrm{~cm}$ telescope. The photopolarimeter works by amplifying the direct current. It can be used as a photoelectric photometer when the linear polarizing filter (polaroid) is removed. The maximum sensitivity of the said photomultiplier lies in the wavelength range $4000-4400 \AA$. The observations were performed in the BVR bands of the spectrum. The uncertainties in photometric and polarimetric measurements respectively are:

$$
\sigma_{m(B V R)}=0 .{ }^{\mathrm{m}} 01-0.02 ; \quad \sigma_{P \%(B V R)}^{\mathrm{m}}=0.02 \% \quad \text { and } \quad \sigma_{\theta(B V R)}=2^{\circ}
$$

\section{Results}

During 21 nights, 30 photometric and polarimetric measurements were performed in the BVR bands. The results of those observations have shown that at the constant brightness of the star, strong changes of light polarization in all three bands of the spectrum were detected. Some changes of the polarization angle have been detected also. This is the first time that such a result for a post-AGB star has been detected. More detailed and multilateral observations in the future could help us in the explanation of this phenomenon.

\section{References}

Melikian N.D., Magnan C., Eritsian M.A., Karapetian A.A., 2000, Astrofizika, 43, 581 Melikian N.D., Della Valle M., \& Natsvlishvili R.Sh., 1988, Astrofizika, 28, 329 Melikian N.D., Jakubov S.D., 1995, Astrofizika, 38, 5 